Article

\title{
Some Results on the Symmetric Representation of the Generalized Drazin Inverse in a Banach Algebra
}

\author{
Yonghui Qin ${ }^{1}$, Xiaoji Liu ${ }^{2, *}$ and Julio Benítez ${ }^{3, *}$ \\ 1 College of Mathematics and Computing Science, Guilin University of Electronic Technology, Guilin 541004, \\ China; yonghui1676@163.com \\ 2 College of Mathematics and Computer Science, Guangxi University for Nationalities, \\ Nanning 530006, China \\ 3 Department of Applied Mathematics, Universitat Politècnica de València, 46022 Valencia, Spain \\ * Correspondence: xiaojiliu72@126.com (X.L.); jbenitez@mat.upv.es (J.B.)
}

Received: 1 January 2019; Accepted: 13 January 2019; Published: 17 January 2019

check for updates

\begin{abstract}
Based on the conditions $a b^{2}=0$ and $b^{\pi}(a b) \in \mathscr{A}^{\mathrm{d}}$, we derive that $(a b)^{n},(b a)^{n}$, and $a b+b a$ are all generalized Drazin invertible in a Banach algebra $\mathscr{A}$, where $n \in \mathbb{N}$ and $a$ and $b$ are elements of $\mathscr{A}$. By using these results, some results on the symmetry representations for the generalized Drazin inverse of $a b+b a$ are given. We also consider that additive properties for the generalized Drazin inverse of the sum $a+b$.
\end{abstract}

Keywords: generalized Drazin inverse; Banach algebra; representation

MSC: 46H05; 47A05; 15A09

\section{Introduction}

Let $\mathscr{A}$ be a complex unital Banach algebra with unit 1 . The sets of all invertible elements and quasinilpotent elements of $\mathscr{A}$ are denoted by $\mathscr{A}^{-1}$ and $\mathscr{A}^{\text {qnil }}$, respectively, where $\mathscr{A}^{-1}:=\left\{a \in \mathscr{A}^{-}\right.$ $\exists x \in \mathscr{A}: a x=x a=1\}$ and $\mathscr{A}^{\text {qnil }}:=\left\{a \in \mathscr{A}: \lim _{n \rightarrow+\infty}\left\|a^{n}\right\|^{1 / n}=0\right\}$. Let $a \in \mathscr{A}$ and, if there is a element $b \in \mathscr{A}$ such that

$$
b a b=b, \quad a b=b a, \text { and } \quad a(1-a b) \text { is quasinilpotent, }
$$

then $b$ is the generalized Drazin inverse of $a$, denoted by $a^{\mathrm{d}}$, and it is unique. The set of generalized Drazin-invertible elements is denoted by $\mathscr{A}^{\mathrm{d}}=\left\{a \in \mathscr{A}: \exists a^{\mathrm{d}}\right\}$. In particular, if $a(1-a b)=0$ (or $a b a=a)$, then $b$ is called the group inverse of $a$. Note that $a a^{\mathrm{d}}$ is an idempotent element and let $a^{\pi}=1-a a^{\mathrm{d}}$. It was given, in [1] (Lemma 2.4), that $a^{\mathrm{d}}$ exists if and only if there is an idempotent $q \in \mathscr{A}$, such that $a q=q a, a q$ is quasinilpotent, and $a+q$ is invertible.

The generalized inverse in a matrix or operator theory is very useful in scientific calculation and in various engineering technologies [2-4]. It is well known that the Drazin inverse has been applied in a few fields, such as statistics and probability [5], ordinary differential equations [6], Markov chains [7], operator matrices [8], neural network models [9,10], and the references therein. In [11], a study of the Drazin inverse for bounded linear operators in a Banach space $X$ is given, when 0 is an isolated spectral point of the operator. In [12], some additive results on the Drazin inverse, under the condition $a b=0$, are obtained. However, as in $[12,13]$, this condition was not enough to derive a formula for the generalized Drazin inverse for $a+b$. In [14], authors investigated how to express the Drazin inverse of sums, differences, and products of two matrices $P$ and $Q$, under the conditions $P^{3} Q=Q P$ and $Q^{3} P=P Q$. The representations of the Drazin inverse for $(P+Q)$, such that $P Q P=0$ and $P Q^{2}=0$, is 
given in [15]. The generalized inverses in $C^{*}$-algebras has been investigated in [16] and a symmetry of the generalized Drazin inverse in a $C^{*}$-algebra has been considered in [17].

Some additive properties of the generalized Drazin inverse in a Banach algebra were investigated in [18]. Recently, the expression for the generalized Drazin inverse of the sum $a+b$ on Banach algebra has been studied, such as in the representations of the generalized Drazin inverse for $a+b$ in a Banach algebra, obtained in [19]; some new additive results for the generalized Drazin inverse in a Banach algebra, given in [20]; and additive results on the generalized Drazin inverse of a sum of two elements in a Banach algebra are derived in [21] and the references therein. In this paper, we consider the representations of the generalized Drazin inverse of the sum of two elements in a Banach algebra. By using the assumed conditions $a b^{2}=0$ and $b^{\pi}(a b) \in \mathscr{A}^{\mathrm{d}}$, it is implied that $(a b)^{n},(b a)^{n}$, and $a b+b a \in \mathscr{A}^{\mathrm{d}}$, and a symmetry representation for the generalized Drazin inverse of $a b+b a$ is obtained, where $n \in \mathbb{N}$ and $a, b \in \mathscr{A}^{\mathrm{d}}$. We also consider the additive properties for the generalized Drazin inverse of the sum $a+b$.

In Section 2, some notation is introduced and lemmas are given. In Section 3, a symmetric representation of the generalized Drazin inverse for $a b+b a$ in a Banach algebra is derived. The additive properties of the generalized Drazin inverse of $a+b$ are investigated in Section 4 .

\section{Preliminaries}

Let $\mathscr{B}$ be a subalgebra of the unital algebra $\mathscr{A}$. For an element $b \in \mathscr{B}^{-1}$, the inverse of $b$ in $\mathscr{B}$ is denoted by $\left[b^{-1}\right]_{\mathscr{B}}$. As in [19], it is given that $\mathscr{B}^{-1} \not \subset \mathscr{A}^{-1}$. Let $\mathscr{P}=\left\{p_{1}, p_{2}, \ldots, p_{n}\right\}$ be a total system of idempotents in $\mathscr{A}$ if $p_{i}^{2}=p_{i}$, for all $i, p_{i} p_{j}=0$ if $i \neq j$, and $p_{1}+\cdots+p_{n}=1$, as in [22]. If $a \in \mathscr{A}^{\mathrm{d}}$, then

$$
a=\left[\begin{array}{cc}
a_{1} & 0 \\
0 & a_{2}
\end{array}\right]_{\mathscr{P}}, \quad a^{\mathrm{d}}=\left[\begin{array}{cc}
{\left[a_{1}^{-1}\right]_{p \mathscr{A} p}} & 0 \\
0 & 0
\end{array}\right]_{\mathscr{P}}, \quad a^{\pi}=\left[\begin{array}{cc}
0 & 0 \\
0 & 1-p
\end{array}\right]_{\mathscr{P}},
$$

where $p=a a^{\mathrm{d}}, \mathscr{P}=\{p, 1-p\}, a_{1} \in[p \mathscr{A} p]^{-1}$, and $a_{2} \in[(1-p) \mathscr{A}(1-p)]^{\text {qnil. }}$ If $a$ has the representation given as in (2), then $a^{\mathrm{d}}=\left[a_{1}^{-1}\right]_{p \mathscr{A} p}=a_{1}^{\mathrm{d}}$.

The following lemmas are required in what follows.

Lemma 1 ([19]). Let $\mathscr{P}=\{p, 1-p\}$ be a total system of idempotents in $\mathscr{A}$, and let $a, b \in \mathscr{A}$ have the following representation

$$
a=\left[\begin{array}{ll}
x & 0 \\
z & y
\end{array}\right]_{\mathscr{P}} \quad b=\left[\begin{array}{ll}
x & t \\
0 & y
\end{array}\right]_{\mathscr{P}} .
$$

Then there exist $\left(z_{n}\right)_{n=0}^{\infty} \subset(1-p) \mathscr{A} p$ and $\left(t_{n}\right)_{n=0}^{\infty} \subset p \mathscr{A}(1-p)$, such that

$$
a^{n}=\left[\begin{array}{cc}
x^{n} & 0 \\
z_{n} & y^{n}
\end{array}\right]_{\mathscr{P}}, \quad b^{n}=\left[\begin{array}{cc}
x^{n} & t_{n} \\
0 & y^{n}
\end{array}\right]_{\mathscr{P}}, \quad \forall n \in \mathbb{N} .
$$

Lemma 2 ([22]). Let $a, b \in \mathscr{A}$ be generalized Drazin invertible and $a b=0$. Then, $a+b$ is generalized Drazin invertible and

$$
(a+b)^{\mathrm{d}}=b^{\pi} \sum_{n=0}^{\infty} b^{n}\left(a^{\mathrm{d}}\right)^{n+1}+\sum_{n=0}^{\infty}\left(b^{\mathrm{d}}\right)^{n+1} a^{n} a^{\pi} .
$$

Lemma 3 ([22]). Let $x, y \in \mathscr{A}, p$ be an idempotent of $\mathscr{A}$, and let $x$ and $y$ have the representation

$$
x=\left[\begin{array}{ll}
a & 0 \\
c & b
\end{array}\right]_{\{p, 1-p\}}, \quad y=\left[\begin{array}{ll}
b & c \\
0 & a
\end{array}\right]_{\{1-p, p\}} .
$$


(i) If $a \in[p \mathscr{A} p]^{\mathrm{d}}$ and $b \in[(1-p) \mathscr{A}(1-p)]^{\mathrm{d}}$, then $x, y \in \mathscr{A}^{\mathrm{d}}$ and

$$
x^{\mathrm{d}}=\left[\begin{array}{cc}
a^{\mathrm{d}} & 0 \\
u & b^{\mathrm{d}}
\end{array}\right]_{\{p, 1-p\}}, \quad y^{\mathrm{d}}=\left[\begin{array}{cc}
b^{\mathrm{d}} & u \\
0 & a^{\mathrm{d}}
\end{array}\right]_{\{1-p, p\}},
$$

where

$$
u=\sum_{n=0}^{\infty}\left(b^{\mathrm{d}}\right)^{n+2} c a^{n} a^{\pi}+\sum_{n=0}^{\infty} b^{\pi} b^{n} c\left(a^{\mathrm{d}}\right)^{n+2}-b^{\mathrm{d}} c a^{\mathrm{d}} .
$$

(ii) If $x \in \mathscr{A}^{\mathrm{d}}$ and $a \in[p \mathscr{A} p]^{\mathrm{d}}$, then $b \in[(1-p) \mathscr{A}(1-p)]^{\mathrm{d}}$, and $x^{\mathrm{d}}$ and $y^{\mathrm{d}}$ are given by (4) and (5).

Lemma 4 ([11]). Let $a \in \mathscr{A}^{\mathrm{d}}$. Then $\left[(a)^{n}\right]^{\mathrm{d}}=\left[a^{\mathrm{d}}\right]^{n}$, for all $n=1,2, \cdots$.

Lemma 5 ([11]). If $a, b \in \mathscr{A}^{\mathrm{d}}$ and $a b=b a=0$. Then, $(a+b)^{\mathrm{d}}$ also exists and $(a+b)^{\mathrm{d}}=a^{\mathrm{d}}+b^{\mathrm{d}}$.

Lemma 6 ([23]). Let $a, b \in \mathscr{A}^{\mathrm{d}}$. Then $(a b)^{n+1}$ is generalized Drazin invertible, for some $n \in \mathbb{N}$, if and only if $a b$ is generalized Drazin invertible.

Lemma 7 ([23]). Let $a, b \in \mathscr{A}^{\mathrm{d}}$ and $(a b)^{n+1}$ be generalized Drazin invertible for some $n \in \mathbb{N}$. Then, $(b a)^{n}$ is generalized Drazin invertible and $\left[(b a)^{n}\right]^{\mathrm{d}}=b\left[(a b)^{n+1}\right]^{\mathrm{d}} a$.

\section{The Symmetric Representation for the Generalized Drazin Inverse of $a b+b a$}

Let $a, b \in \mathscr{A}^{\mathrm{d}}$. A symmetric expression of $(a b+b a)^{\mathrm{d}}$ is given, by using $a b, b a,(a b)^{\mathrm{d}}$, and $(b a)^{\mathrm{d}}$, with the following assumed conditions

$$
a b^{2}=0, \quad b^{\pi}(a b) \in \mathscr{A}^{\mathrm{d}} .
$$

Theorem 1. Let $a, b \in \mathscr{A}^{\mathrm{d}}$ satisfy (6). Then, $(a b)^{n},(b a)^{n}, a b+b a \in \mathscr{A}^{\mathrm{d}}(n=1,2, \cdots)$, and a representation of $(a b+b a)^{\mathrm{d}}$ is given as

$$
(a b+b a)^{\mathrm{d}}=(b a)^{\pi} \sum_{n=1}^{\infty}(b a)^{n-1}\left[(a b)^{n}\right]^{\mathrm{d}}+\sum_{n=1}^{\infty}\left[(b a)^{n}\right]^{\mathrm{d}}(a b)^{n-1}(a b)^{\pi} .
$$

Proof. Let $b=\left[\begin{array}{cc}b_{1} & 0 \\ 0 & b_{2}\end{array}\right]_{\mathscr{P}}$, where $\mathscr{P}=\left\{b b^{\mathrm{d}}, b^{\pi}\right\}, b_{1}$ is invertible in the subalgebra $b b^{\mathrm{d}} \mathscr{A} b b^{\mathrm{d}}$, and $b_{2}$ is quasinilpotent. Let us write $a=\left[\begin{array}{ll}a_{11} & a_{12} \\ a_{21} & a_{22}\end{array}\right]_{\mathscr{P}}$. From $a b^{2}=0$, we have

$$
a_{11}=0, \quad a_{21}=0, \quad a_{12} b_{2}^{2}=0, \quad \text { and } \quad a_{22} b_{2}^{2}=0 .
$$

Thus, we have $a b=\left[\begin{array}{ll}0 & a_{12} b_{2} \\ 0 & a_{22} b_{2}\end{array}\right]$. By Lemma 3, we obtain that $a b \in \mathscr{A}^{\mathrm{d}}$ if and only if $a_{22} b_{2}$ is generalized Drazin invertible. Thus, $\left(b^{\pi} a b\right)^{\mathrm{d}}$ exists. By using Cline's formula, it proves that $(a b)^{\mathrm{d}}$ also is. Therefore, we obtain $(a b)^{n},(b a)^{n} \in \mathscr{A}^{\mathrm{d}}$ by using Lemma 6 and 7. Since $a b^{2}=0$, by Lemma 2 we can prove that $a b+b a$ is generalized Drazin invertible and that (7) holds. If $n=1$, then $(a b+b a)^{\mathrm{d}}=$ $(b a)^{\pi}(a b)^{\mathrm{d}}+(b a)^{\mathrm{d}}(a b)^{\pi}$. By using mathematical induction, we derive that the representation can be given, as in (7).

Remark 1. Note that the expression given in Theorem 1 is symmetric. 
Theorem 2. Let $a, b \in \mathscr{A}^{\mathrm{d}}$ satisfy (6) and $a^{2}=0$. Then $a b+b a \in \mathscr{A}^{\mathrm{d}}$ and $\left[(a b+b a)^{\mathrm{d}}\right]^{n}=\left[(a b)^{\mathrm{d}}\right]^{n}+$ $\left[(b a)^{\mathrm{d}}\right]^{n}$, for all $n=1,2, \cdots$.

Proof. Let $a, b$ be written as in the proof of Theorem 1 , and, by $a b^{2}=0$, we derive $a b=\left[\begin{array}{ll}0 & a_{12} b_{2} \\ 0 & a_{22} b_{2}\end{array}\right]$ and $a b, b a,(a b)^{n},(b a)^{n} \in \mathscr{A}^{\mathrm{d}}$. Since $a b^{2}=0$ and $a^{2}=0$, we have

$$
(a b)^{n}(b a)^{n}=(b a)^{n}(a b)^{n}=0, \quad(a b+b a)^{n}=(a b)^{n}+(b a)^{n},
$$

for all $n=1,2, \cdots$. By Lemma 4, Lemma 5 , and the first equality of (9), we derive

$$
\left[(a b+b a)^{\mathrm{d}}\right]^{n}=\left[(a b+b a)^{n}\right]^{\mathrm{d}}=\left[(a b)^{n}+(b a)^{n}\right]^{\mathrm{d}}=\left[(a b)^{n}\right]^{\mathrm{d}}+\left[(b a)^{n}\right]^{\mathrm{d}}=\left[(a b)^{\mathrm{d}}\right]^{n}+\left[(b a)^{\mathrm{d}}\right]^{n} .
$$

At the end of Section 3, let $\mathscr{A}$ be a $C^{*}$-algebra, as in [17]. Then, a simple application of the generalized Drazin inverse in a $C^{*}$-algebra can be given, as follows.

Theorem 3. Let $a, b \in \mathscr{A}$ be group invertible. If (6) is satisfied, then $(a b+b a)^{\dagger}$ exists.

Proof. By using Theorem 1, we derive that $a b+b a$ is group invertible. As pointed out in [16], $a b+b a$ is generalized invertible. Thus, $(a b+b a)^{\dagger}$ exists.

Theorem 4. Let $a, b \in \mathscr{A}^{\mathrm{d}}$. If $(6)$ is satisfied, then $(a b+b a)^{\mathrm{d}}$ is self-adjoint in a $C^{*}$-algebra.

Proof. Note that $a b+b a$ is self-adjoint in a $C^{*}$-algebra. By Theorem 1 and using [17] (Theorem 3.2), we obtain that $(a b+b a)^{\mathrm{d}}$ is self-adjoint in a $C^{*}$-algebra.

\section{The Representation for the Generalized Drazin Inverse of $a+b$}

In this section, we consider some results on the expression of $(a+b)^{\mathrm{d}}$, by using $a, b, a^{\mathrm{d}}$, and $b^{\mathrm{d}}$, where $a, b \in \mathscr{A}^{\mathrm{d}}$.

Lemma 8. Let $a, b \in \mathscr{A}^{\mathrm{d}}$ satisfy $a b^{2}=0$. Then, $(a+b)^{\mathrm{d}}$ exists if and only if $b^{\pi}(a+b) \in \mathscr{A}^{\mathrm{d}}$.

Proof. Similarly, we rewrite $a, b$ as in the proof of Theorem 1 . Since $a b^{2}=0$, we derive

$$
a+b=\left[\begin{array}{cc}
b_{1} & a_{12} \\
0 & b_{2}+a_{22}
\end{array}\right]_{\mathscr{P}} .
$$

By Lemma 3, note that $(a+b)^{\mathrm{d}}$ exists if and only if $\left(a_{22}+b_{2}\right)^{\mathrm{d}}$ exists; that is, $(a+b)^{\mathrm{d}}$ exists if and only if $b^{\pi}(a+b)$ is generalized Drazin invertible.

Theorem 5. Let $a, b \in \mathscr{A}^{\mathrm{d}}$ satisfy the conditions of Theorem 2. Then

$$
\begin{aligned}
(a+b)^{\mathrm{d}} & =\sum_{n=0}^{\infty}\left(b^{\mathrm{d}}\right)^{2 n+1}\left[b^{\mathrm{d}}(a b)^{\pi}(a b)^{n} a+(a b)^{\pi}(a b)^{n}\right] \\
& -\sum_{n=0}^{\infty} b^{\pi} b^{2 n}\left\{\left[(a b)^{\mathrm{d}}\right]^{n+1} a+b\left[(a b)^{\mathrm{d}}\right]^{n+1}\right\} .
\end{aligned}
$$

Proof. By Lemma 8, it also leads to (10). By Lemma 3, we can prove that $(a+b)^{\mathrm{d}}$ exists if and only if $\left(a_{22}+b_{2}\right)^{\mathrm{d}}$ exists; that is, $(a+b)^{\mathrm{d}}$ exists if and only if $b^{\pi}(a+b)$ is generalized Drazin invertible. 
If $b^{\pi} a b \in \mathscr{A}^{\mathrm{d}}$, then $\left(a_{22} b_{2}\right)^{\mathrm{d}}$ exists. By Cline's formula, we have that $\left(b_{2} a_{22}\right)^{\mathrm{d}}$ exists. As in the proof of Theorem 1, by Lemma 6 and 7 , we also obtain that $(a b)^{n},(b a)^{n} \in \mathscr{A}^{\mathrm{d}}$, for all $n=1,2, \cdots$.

By $a^{2}=0$, we get

$$
a_{12} a_{22}=0 \quad \text { and } \quad a_{22}^{2}=0 .
$$

By (8) and (11), we have $\left(b_{2} a_{22}\right)\left(a_{22} b_{2}\right)=0,\left(a_{22} b_{2}\right)\left(b_{2} a_{22}\right)=0$. Using Lemma 5 , and by Cline's formula, we derive

$$
\left(a_{22} b_{2}+b_{2} a_{22}\right)^{\mathrm{d}}=\left(a_{22} b_{2}\right)^{\mathrm{d}}+\left(b_{2} a_{22}\right)^{\mathrm{d}} .
$$

By induction, let $\left[\left(a_{22} b_{2}\right)^{\mathrm{d}}+\left(b_{2} a_{22}\right)^{\mathrm{d}}\right]^{n}=\left[\left(a_{22} b_{2}\right)^{\mathrm{d}}\right]^{n}+\left[\left(b_{2} a_{22}\right)^{\mathrm{d}}\right]^{n}$ for all $n \geq 1$. Therefore, we can prove that

$$
\left[\left(a_{22} b_{2}+b_{2} a_{22}\right)^{\mathrm{d}}\right]\left[\left(a_{22} b_{2}\right)^{\mathrm{d}}+\left(b_{2} a_{22}\right)^{\mathrm{d}}\right]^{n}=\left[\left(a_{22} b_{2}\right)^{\mathrm{d}}\right]^{n+1}+\left[\left(b_{2} a_{22}\right)^{\mathrm{d}}\right]^{n+1} .
$$

Since $\left(a_{22} b_{2}+b_{2} a_{22}\right) b_{2}^{2}=0$ and $b_{2}$ are quasinilpotent, by Lemma 5 and (12), we obtain

$$
\begin{aligned}
{\left[\left(a_{22}+b_{2}\right)^{2}\right]^{\mathrm{d}} } & =\left(a_{22} b_{2}+b_{2} a_{22}+b_{2}^{2}\right)^{\mathrm{d}} \\
& =\sum_{n=0}^{\infty} b_{2}^{2 n}\left[\left(a_{22} b_{2}+b_{2} a_{22}\right)^{\mathrm{d}}\right]^{n+1} \\
& =\sum_{n=0}^{\infty} b_{2}^{2 n}\left[\left(a_{22} b_{2}\right)^{\mathrm{d}}+\left(b_{2} a_{22}\right)^{\mathrm{d}}\right]^{n+1} .
\end{aligned}
$$

Then, $b^{\pi}(a+b) \in \mathscr{A}^{\mathrm{d}}$ implies that $\left(a_{22}+b_{2}\right)^{\mathrm{d}}$ exists and $\left(a_{22}+b_{2}\right)^{\mathrm{d}}=\left[\left(a_{22}+b_{2}\right)^{2}\right]^{\mathrm{d}}\left(a_{22}+b_{2}\right)$. Finally, by (13), and $\left(b_{2} a_{22}\right)^{\mathrm{d}}=b_{2}\left[\left(a_{22} b_{2}\right)^{\mathrm{d}}\right]^{2} a_{22}$, we obtain

$$
\begin{aligned}
\left(a_{22}+b_{2}\right)^{\mathrm{d}} & =\left[\left(a_{22}+b_{2}\right)^{\mathrm{d}}\right]^{2}\left(a_{22}+b_{2}\right) \\
& =\sum_{n=0}^{\infty} b_{2}^{2 n}\left\{\left[\left(a_{22} b_{2}\right)^{\mathrm{d}}\right]^{n+1}+\left(b_{2}\left[\left(a_{22} b_{2}\right)^{\mathrm{d}}\right]^{2} a_{22}\right)^{n+1}\right\}\left(a_{22}+b_{2}\right) \\
& =\sum_{n=0}^{\infty} b_{2}^{2 n}\left[\left(a_{22} b_{2}\right)^{\mathrm{d}}\right]^{n+1} a_{22}+\sum_{n=0}^{\infty} b_{2}^{2 n}\left[b_{2}\left(\left(a_{22} b_{2}\right)^{\mathrm{d}}\right)^{2} a_{22}\right]^{n+1} b_{2} \\
& =\sum_{n=0}^{\infty} b_{2}^{2 n}\left\{\left[\left(a_{22} b_{2}\right)^{\mathrm{d}}\right]^{n+1} a_{22}+b_{2}\left[\left(a_{22} b_{2}\right)^{\mathrm{d}}\right]^{n+1}\right\}
\end{aligned}
$$

and

$$
\left(a_{22}+b_{2}\right)^{\pi}=\left(a_{22} b_{2}\right)^{\pi}-\sum_{n=0}^{\infty} b_{2}^{2 n+1}\left\{\left[\left(a_{22} b_{2}\right)^{\mathrm{d}}\right]^{n+1} a_{22}+b_{2}\left[\left(a_{22} b_{2}\right)^{\mathrm{d}}\right]^{n+1}\right\}
$$

By Lemma 3, we get that $a+b \in \mathscr{A}^{\mathrm{d}}$ and

$$
(a+b)^{\mathrm{d}}=\left[\begin{array}{cc}
b_{1}^{-1} & u \\
0 & \left(a_{22}+b_{2}\right)^{\mathrm{d}}
\end{array}\right]_{\mathscr{P}},
$$

and

$$
u=\sum_{n=0}^{\infty}\left(b_{1}^{-1}\right)^{n+2} a_{12}\left(b_{2}+a_{22}\right)^{n}\left(a_{22}+b_{2}\right)^{\pi}-\left(a_{22}+b_{2}\right)^{\mathrm{d}} a_{12} b_{1}^{-1} .
$$


Evidently, we have $\left[b_{1}^{-1}\right]_{\mathscr{P}}=b^{\mathrm{d}}$ and

$$
b^{\mathrm{d}} b a=\left[\begin{array}{cc}
b_{1}^{-1} b_{1} & 0 \\
0 & 0
\end{array}\right]_{\mathscr{P}}\left[\begin{array}{ll}
0 & a_{12} \\
0 & a_{22}
\end{array}\right]_{\mathscr{P}}=\left[\begin{array}{cc}
0 & a_{12} \\
0 & 0
\end{array}\right]_{\mathscr{P}}=a_{12}
$$

One easily has (by induction and by using (8) and (11)) that, if $n \geq 1$, then

$$
a_{12}\left(a_{22}+b_{2}\right)^{n}= \begin{cases}a_{12}\left(b_{2} a_{22}\right)^{n / 2} & \text { if } n \text { is even, } \\ a_{12}\left(b_{2} a_{22}\right)^{(n-1) / 2} b_{2} & \text { if } n \text { is odd. }\end{cases}
$$

By Lemma 1, we obtain that, for any $n \geq 1$,

$$
b^{\pi}(b a)^{n}=\left[\begin{array}{cc}
0 & 0 \\
0 & b^{\pi}
\end{array}\right]_{\mathscr{P}}\left[\begin{array}{cc}
0 & x_{n} \\
0 & \left(b_{2} a_{22}\right)^{n}
\end{array}\right]_{\mathscr{P}}=\left[\begin{array}{cc}
0 & 0 \\
0 & \left(b_{2} a_{22}\right)^{n}
\end{array}\right]_{\mathscr{P}}=\left(b_{2} a_{22}\right)^{n},
$$

where $\left(x_{n}\right)_{n=0}^{\infty}$ is a sequence in $\mathscr{A}$. Furthermore, one has $b_{2}=b^{\pi} b=b b^{\pi}$ and $a b^{\pi}=a\left(1-b b^{\mathrm{d}}\right)=$ $a\left(1-b^{2}\left(b^{\mathrm{d}}\right)^{2}\right)=a$. Hence, if $n \geq 1$ is even, then

$$
a_{12}\left(a_{22}+b_{2}\right)^{n}=a_{12}\left(b_{2} a_{22}\right)^{n / 2}=b^{\mathrm{d}} b a b^{\pi}(b a)^{n / 2}=b^{\mathrm{d}} b a(b a)^{n / 2}=b^{\mathrm{d}}(b a)^{(n+2) / 2},
$$

and if $n \geq 1$ is odd, then

$$
a_{12}\left(a_{22}+b_{2}\right)^{n}=a_{12}\left(b_{2} a_{22}\right)^{(n-1) / 2} b_{2}=b^{\mathrm{d}} b a b^{\pi}(b a)^{(n-1) / 2} b^{\pi} b=b^{\mathrm{d}}(b a)^{(n+1) / 2} b .
$$

From (15), we have

$$
\begin{aligned}
& a_{12}\left(a_{22}+b_{2}\right)^{\pi}=a_{12}\left(1-b_{2}\left(a_{22} b_{2}\right)^{\mathrm{d}} a_{22}\right), \\
& a_{22}\left(a_{22}+b_{2}\right)^{\pi}=\left(a_{22} b_{2}\right)^{\pi} a_{22}, \\
& a_{12} b_{2}\left(a_{22}+b_{2}\right)^{\pi}=a_{12} b_{2}\left(a_{22} b_{2}\right)^{\pi}, \\
& a_{22} b_{2}\left(a_{22}+b_{2}\right)^{\pi}=a_{22} b_{2}\left(a_{22} b_{2}\right)^{\pi} .
\end{aligned}
$$

Thus, by using the obvious equality $(b a)^{k} b=b(a b)^{k}$, and by (14)-(16) and (18), we have

$$
\begin{gathered}
(a+b)^{\mathrm{d}}=b_{1}^{\mathrm{d}}+u=\left[b_{1}\right]_{\mathscr{P}}^{-1}+\sum_{n=0}^{\infty}\left(\left[b_{1}^{-1}\right]_{\mathscr{P}}\right)^{n+2} a_{12}\left(b_{2}+a_{22}\right)^{n}\left(a_{22}+b_{2}\right)^{\pi} \\
-\left(a_{22}+b_{2}\right)^{\mathrm{d}} a_{12} b_{1}^{-1}+\left(a_{22}+b_{2}\right)^{\mathrm{d}} \\
=\sum_{n=0}^{\infty}\left(b^{\mathrm{d}}\right)^{2 n+2} b^{\pi}(a b)^{n} a+\sum_{n=0}^{\infty}\left(b^{\mathrm{d}}\right)^{2 n+1} b^{\pi}(a b)^{n} \\
-\sum_{n=0}^{\infty} b^{\pi} b^{2 n}\left\{\left[(a b)^{\mathrm{d}}\right]^{n+1} a+b\left[(a b)^{\mathrm{d}}\right]^{n+1}\right\} \\
=\sum_{n=0}^{\infty}\left(b^{\mathrm{d}}\right)^{2 n+1}\left[b^{\mathrm{d}}(a b)^{\pi}(a b)^{n} a+(a b)^{\pi}(a b)^{n}\right] \\
\quad-\sum_{n=0}^{\infty} b^{\pi} b^{2 n}\left\{\left[(a b)^{\mathrm{d}}\right]^{n+1} a+b\left[(a b)^{\mathrm{d}}\right]^{n+1}\right\} .
\end{gathered}
$$

The proof is completed. 
Theorem 6. Let $a, b \in \mathscr{A}^{\mathrm{d}}$ satisfy (6) and $b^{\pi} a^{2}=0$. Then,

$$
(a+b)^{\mathrm{d}}=b^{\mathrm{d}}+u+v
$$

where

$$
\begin{aligned}
& v=-\left\{b^{\mathrm{d}} a(b a)^{\mathrm{d}}+\sum_{n=0}^{\infty} b^{\mathrm{d}} b^{2 n+1}\left[\left((a b)^{\mathrm{d}}\right)^{n+1}+\left((b a)^{\mathrm{d}}\right)^{n+1}\right]\right\} \\
& u=\sum_{n=0}^{\infty}\left(b^{\mathrm{d}}\right)^{n+2} a(a+b)^{n}+\sum_{n=0}^{\infty}\left(1-b^{\pi}\right) b^{n} a v^{n+2}-b^{\mathrm{d}} a v
\end{aligned}
$$

Proof. Let $p=b b^{\mathrm{d}}$ and $\mathscr{P}=\{p, 1-p\}$. Let $a$ and $b$ have the following representation

$$
b=\left[\begin{array}{cc}
b_{1} & 0 \\
0 & b_{2}
\end{array}\right]_{\mathscr{P}}, \quad a=\left[\begin{array}{ll}
a_{3} & a_{1} \\
a_{4} & a_{2}
\end{array}\right]_{\mathscr{P}},
$$

where $b_{1}$ is invertible in $p \mathscr{A} p$ and $b_{2}$ is quasinilpotent in $(1-p) \mathscr{A}(1-p)$. Let us find the expression of $b^{\pi} a^{2}$ in the system of idempotents $\mathscr{P}$ :

$$
b^{\pi} a^{2}=\left[\begin{array}{cc}
0 & 0 \\
0 & 1-p
\end{array}\right]_{\mathscr{P}}\left[\begin{array}{ll}
0 & a_{1} \\
0 & a_{2}
\end{array}\right]_{\mathscr{P}}\left[\begin{array}{ll}
0 & a_{1} \\
0 & a_{2}
\end{array}\right]_{\mathscr{P}}=\left[\begin{array}{cc}
0 & 0 \\
0 & a_{2}^{2}
\end{array}\right]_{\mathscr{P}}=a_{2}^{2} .
$$

Thus, $a_{2}^{2}=0$. On the other hand,

$$
a b^{2}=\left[\begin{array}{ll}
0 & a_{1} \\
0 & a_{2}
\end{array}\right]_{\mathscr{P}}\left[\begin{array}{cc}
b_{1}^{2} & 0 \\
0 & b_{2}^{2}
\end{array}\right]_{\mathscr{P}}=\left[\begin{array}{ll}
0 & a_{1} b_{2}^{2} \\
0 & a_{2} b_{2}^{2}
\end{array}\right]_{\mathscr{P}} .
$$

Therefore, $a_{2} b_{2}^{2}=0$. By $b^{\pi} a b, b^{\pi} b a \in \mathscr{A}^{d}$, we obtain $\left(a_{2} b_{2}\right),\left(b_{2} a_{2}\right) \in \mathscr{A}^{d}$. We can appeal to Theorem 5, obtaining (recall that $b_{2}$ is quasinilpotent and $b_{2}^{\mathrm{d}}=0$ ) that

$$
\left(a_{2}+b_{2}\right)^{\mathrm{d}}=-a_{2}\left(b_{2} a_{2}\right)^{\mathrm{d}}-\sum_{n=0}^{\infty} b_{2}^{2 n+1}\left[\left(\left(a_{2} b_{2}\right)^{\mathrm{d}}\right)^{n+1}+\left(\left(b_{2} a_{2}\right)^{\mathrm{d}}\right)^{n+1}\right] .
$$

From Lemma 3 and the representation of $a+b$ in (16), we have

$$
\begin{aligned}
(a+b)^{\mathrm{d}} & =\left[b_{1}^{-1}\right]_{\mathscr{P}}+\left(a_{2}+b_{2}\right)^{\mathrm{d}}+u \\
& =\left[b_{1}^{-1}\right]_{\mathscr{P}}+u-\left\{a_{2}\left(b_{2} a_{2}\right)^{d}+\sum_{n=0}^{\infty} b_{2}^{2 n+1}\left[\left(\left(a_{2} b_{2}\right)^{d}\right)^{n+1}+\left(\left(b_{2} a_{2}\right)^{d}\right)^{n+1}\right]\right\}
\end{aligned}
$$

where

$$
\begin{aligned}
u & =\sum_{n=0}^{\infty}\left(\left[b_{1}^{-1}\right]_{\mathscr{P}}\right)^{n+2} a_{1}\left(a_{2}+b_{2}\right)^{n}\left(a_{2}+b_{2}\right)^{\pi} \\
& +\sum_{n=0}^{\infty} b_{1}^{\pi} b_{1}^{n} a_{1}\left(\left(a_{2}+b_{2}\right)^{\mathrm{d}}\right)^{n+2}-\left[b_{1}^{-1}\right]_{\mathscr{P}} a_{1}\left(a_{2}+b_{2}\right)^{\mathrm{d}} \\
& =\sum_{n=0}^{\infty}\left(b_{1}^{\mathrm{d}}\right)^{n+2} a_{1}\left(a_{2}+b_{2}\right)^{n} .
\end{aligned}
$$


Observe that $\left[b_{1}^{-1}\right]_{\mathscr{P}}=b^{\mathrm{d}}$, and

$$
\begin{aligned}
\left(b^{\mathrm{d}}\right)^{n+2} a(a+b)^{n} & =\left[\begin{array}{cc}
\left(b_{1}^{\mathrm{d}}\right)^{n+2} & 0 \\
0 & 0
\end{array}\right]_{\mathscr{P}}\left[\begin{array}{ll}
0 & a_{1} \\
0 & a_{2}
\end{array}\right]_{\mathscr{P}}\left[\begin{array}{cc}
b_{1}^{n} & x_{n} \\
0 & \left(a_{2}+b_{2}\right)^{n}
\end{array}\right]_{\mathscr{P}} \\
& =\left[\begin{array}{cc}
0 & 0 \\
0 & \left(b_{1}^{\mathrm{d}}\right)^{n+2} a_{1}\left(a_{2}+b_{2}\right)^{n}
\end{array}\right]_{\mathscr{P}}=\left(b_{1}^{\mathrm{d}}\right)^{n+2} a_{1}\left(a_{2}+b_{2}\right)^{n}, \\
v=b^{\pi}(a+b)^{\mathrm{d}} & =\left(a_{2}+b_{2}\right)^{\mathrm{d}}=-\left\{b^{\mathrm{d}} a(b a)^{\mathrm{d}}+\sum_{n=0}^{\infty} b^{\mathrm{d}} b^{2 n+1}\left[\left((a b)^{\mathrm{d}}\right)^{n+1}+\left((b a)^{\mathrm{d}}\right)^{n+1}\right]\right\} .
\end{aligned}
$$

Thus, the above expression of $u$ reduces to

$$
u=\sum_{n=0}^{\infty}\left(b^{\mathrm{d}}\right)^{n+2} a(a+b)^{n}+\sum_{n=0}^{\infty}\left(1-b^{\pi}\right) b^{n} a(v)^{n+2}-b^{\mathrm{d}} a v .
$$

Expressions (20) and (21) finish the proof.

\section{Conclusions}

In this paper, we have proved that the multiplications $(a b)^{n}$ and $(b a)^{n}$ of elements $a, b \in \mathscr{A}^{\mathrm{d}}$ in a Banach algebra are both generalized Drazin invertible with the conditions (6). A symmetry representation of the generalized Drazin inverse for $a b+b a$ has been derived. The expression given in Theorem 1 is symmetric, as in Remark 1 . In the other words, if the result is applied in the computation of $(a b+b a)^{\mathrm{d}}$, maybe it will improve the corresponding computational effectiveness and reduce its complexity. The additive properties of $(a+b)^{\mathrm{d}}$ have been investigated under the conditions $a b^{2}=0$, $b^{\pi} a b \in \mathscr{A}^{\mathrm{d}}$, and $a^{2}=0$. With similar conditions, but $a^{2}=0$ being replaced by $b^{\pi} a^{2}=0$, we have also given a resulting expression of $(a+b)^{\mathrm{d}}$.

In fact, as pointed out as in [19], it is still an interesting and open problem to express the generalized Drazin inverse of $a+b$ as a function of $a, b$, and their respective generalized Drazin inverses. In the future, we plan to consider the representations of the generalized Drazin inverse for $a \pm b$ by using $a, b$, and their generalized Drazin inverses, without side conditions.

Author Contributions: Funding acquisition, Y.Q. and X.L.; Methodology, X.L.; Supervision, J.B.; Writing-review and editing, Y.Q.

Funding: This work was supported by the National Natural Science Foundation of China (grant number: 11361009, 61772006,11561015), the Special Fund for Science and Technological Bases and Talents of Guangxi (grant number: 2016AD05050, 2018AD19051), the Special Fund for Bagui Scholars of Guangxi (grant number: 2016A17), the High level innovation teams and distinguished scholars in Guangxi Universities (grant number: GUIJIAOREN201642HAO), the Natural Science Foundation of Guangxi(grant number: 2017GXNSFBA198053, 2018JJD110003), and the open fund of Guangxi Key laboratory of hybrid computation and IC design analysis (grant number: HCIC201607).

Conflicts of Interest: The authors declare that they have no conflict of interest.

\section{References}

1. Castro González, N. Additive perturbation results for the Drazin inverse. Linear Algebra Appl. 2005, 397, 279-297. [CrossRef]

2. Ben-Israel, A.; Greville, T.N.E. Generalized Inverses: Theory and Applications; John Wiley \& Sons: New York, NY, USA; London, UK; Sydney, Australia, 1974.

3. Djordjević, D.S.; Rakočević, V. Lectures on Generalized Inverses; Faculty of Sciences and Mathematics, University of Niš: Niš, Serbia, 2008.

4. Wang, G.R.; Wei, Y.M.; Qiao, S.Z. Generalized Inverses: Theory and Computations, 2nd ed.; Developments in Mathematics; Springer: Singapore; Science Press: Beijing, China, 2018; Volume 53. 
5. Campbell, S.L.; Meyer, C.D. Generalized Inverses of Linear Transformations; Classics in Applied Mathematics; Society for Industrial and Applied Mathematics (SIAM): Philadelphia, PA, USA, 2009; Volume 56.

6. Bulatova, A.A. Numerical solution of degenerate systems of second-order ordinary differential equations using the Drazin inverse matrix. In Algebrodifferential Systems and Methods for Their Solution (Russian); VO Nauka: Novosibirsk, Russia, 1993; Volume 90, pp. 28-43.

7. Zhang, X.Y.; Chen, G.L. The computation of Drazin inverse and its application in Markov chains. Appl. Math. Comput. 2006, 183, 292-300. [CrossRef]

8. Castro-González, N.; Dopazo, E.; Martínez-Serrano, M.F. On the Drazin inverse of the sum of two operators and its application to operator matrices. J. Math. Anal. Appl. 2009, 350, 207-215. [CrossRef]

9. Qiao, S.Z.; Wang, X.Z.; Wei, Y.M. Two finite-time convergent Zhang neural network models for time-varying complex matrix Drazin inverse. Linear Algebra Appl. 2018, 542, 101-117. [CrossRef]

10. Stanimirović, P.S.; Živković, I.S.; Wei, Y.M. Recurrent neural network for computing the Drazin inverse. IEEE Trans. Neural Netw. Learn. Syst. 2015, 26, 2830-2843. [CrossRef] [PubMed]

11. Koliha, J.J. A generalized Drazin inverse. Glasg. Math. J. 1996, 38, 367-381. [CrossRef]

12. Hartwig, R.E.; Wang, G.R.; Wei, Y.M. Some additive results on Drazin inverse. Linear Algebra Appl. 2001, 322, 207-217. [CrossRef]

13. Djordjević, D.S.; Wei, Y.M. Additive results for the generalized Drazin inverse. J. Aust. Math. Soc. 2002, 73, 115-125. [CrossRef]

14. Liu, X.J.; Xu, L.; Yu, Y.M. The representations of the Drazin inverse of differences of two matrices. Appl. Math. Comput. 2010, 216, 3652-3661. [CrossRef]

15. Yang, H.; Liu, X.F. The Drazin inverse of the sum of two matrices and its applications. J. Comput. Appl. Math. 2011, 235, 1412-1417. [CrossRef]

16. Harte, R.; Mbekhta, M. On generalized inverses in $C^{*}$-algebras. Stud. Math. 1992, 103, 71-77. [CrossRef]

17. Djordjević, D.S.; Stanimirović, P.S. On the generalized Drazin inverse and generalized resolvent. Czechoslov. Math. J. 2001, 51, 617-634. [CrossRef]

18. Cvetković-Ilić, D.S.; Djordjević, D.S.; Wei, Y. Additive results for the generalized Drazin inverse in a Banach algebra. Linear Algebra Appl. 2006, 418, 53-61. [CrossRef]

19. Benítez, J.; Liu, X.; Qin, Y. Representations for the generalized Drazin inverse in a Banach algebra. Bull. Math. Anal. Appl. 2013, 5, 53-64.

20. Liu, X.J.; Qin, X.L.; Benítez, J. New additive results for the generalized Drazin inverse in a Banach algebra. Filomat 2016, 30, 2289-2294. [CrossRef]

21. Mosić, D.; Zou, H.L.; Chen, J.L. The generalized Drazin inverse of the sum in a Banach algebra. Ann. Funct. Anal. 2017, 8, 90-105. [CrossRef]

22. Castro González, N.; Koliha, J.J. New additive results for the g-Drazin inverse. Proc. R. Soc. Edinb. Sect. A 2004, 134, 1085-1097. [CrossRef]

23. Mosić, D. A note on Cline's formula for the generalized Drazin inverse. Linear Multilinear Algebra 2015, 63, 1106-1110. [CrossRef]

(C) 2019 by the authors. Licensee MDPI, Basel, Switzerland. This article is an open access article distributed under the terms and conditions of the Creative Commons Attribution (CC BY) license (http://creativecommons.org/licenses/by/4.0/). 